\title{
OUTCOME OF PCI IN PATIENTS WITH LV DYSFUNCTION
}

\author{
Asha Mahilamaran
}

Impact of LV dysfunction on mortality following PCI has been reported in several retrospective and prospective analysis. There are no randomized trial data comparing outcomes in patients undergoing CABG or PCI as compared to optimal medical treatment in patients with LV dysfunction. The SYNTAX and FREEDOM trial have included less than 3\% of patients with LV dysfunction. In many PCI trials assessment of LV dysfunction is present in less than half of the patients. The analysis of outcomes of PCI in patients with LV dysfunction is dependent on registry data and many of them in the last decade may not be relevant today with overall improved hardware and techniques resulting in greater safety of the procedure.

In this issue the article on Outcome after PCI in patients with LV dysfunction insignificant number- 329 patients with LV dysfunction in the contemporary PCI scenario is relevant. The data is very assuring with across the spectrum of PCI in stable, NSTEMI and STEMI the one year MACCE rate is low and comparable to good LV function, with only severe LV dysfunction having worse outcomes. But they have not differentiated in-hospital versus follow-up events in the various groups and also no mention of haemodynamic support devices in the high risk group has been analysed to assist in guiding future understanding and management in the high risk group. The numbers are very low for meaningful conclusions in very high risk subset and analysis of larger database and registry with a special focus on these issues may throw some light.

A meta-analysis of 19 studies with outcome measures in PCI with impaired LV systolic dysfunction by Kunnadian et al In-hospital mortality was acceptable at $18 \%$, with long term mortality of $15.6 \%$ [1].

Article received on 01 APR 2017, published on 30 APR 2017

Asha Mahilmaran ${ }^{1}$

${ }^{1}$ Senior Interventional Consultant Cardiologist, Apollo Hospitals,

Chennai, India.

Corresponding Author: Asha Mahilmaran

Email: ashamahil@hotmail.com
The major predictors of outcomes in PCI with LV dysfunction depend on an assessment of myocardial viability, patients with viability only having survival. In the study by Ammirati et al [2], a total of 385 patients, $36 \%$ underwent stress testing, mostly SPECT.LVEF $<35 \%$ was associated with HR-2.04 for mortality. Assessment of viability was associated with better outcomes. In the study by Daubert et al, high risk PCI was associated with reverse remodeling and $51 \%$ had improvement of LVEF by $13 \%$ and those with LVEF improvement had better outcomes on follow-up [3].

In a Korean study of 329 patients with ACS between 2001-2006, age, $\mathrm{LVEF}<30 \%$, serum creatinine $>3.0 \mathrm{mg} / \mathrm{dl}$, female gender had worse outcomes.

Use of haemodynamic support devices may improve outcomes in high risk PCI in patients with severe LV dysfunction, MVD, lone surviving vessel, left main or graft interventions. In the BCIS - 1 study( Balloon pump assisted coronary intervention study, in patients with EF $<30 \%, 34 \%$ long termall cause mortality reduction was seen in patients with elective IABP use[4].In the PROTECT II trial use of Impella 2.5 had better outcomes than IABP in PCI with severe LV dysfunction [5].Tandem heart by percutaneous insertion has also been used in recent times with better outcome than even Impella 2.5.

The HORIZONS AMI Trial -compared 3 year outcomes after primary $\mathrm{PCI}$ in patients with LVEF $<40 \%$ versus $>40 \% .3$ year mortality was graded from $<30 \%$ with worst outcome mortality of $29.4 \%$ as compared to $2.9 \%$ in LVEF $>60 \%$.

PCI outcomes in the recent past have improved with better hardware, learned skills and improved pharmacotherapy. Severe LV dysfunction continues to portend worser prognosis, which may be improved by use of appropriate haemodynamic support and assessment of myocardial viability prior to the procedure. LVEF assessment should be included in the pre procedural assessment of all PCI patients as it impacts immediate and long term outcomes. 


\section{REFERENCES:}

1. Kunnadian V, Pugh A, Zaman AG,Qiu W Percutaneous intervention among patients with left ventricular systolic dysfunction:A review and metaanalysis of 19 clinical studies. Coronary Artery Dis.2012; 23(7):469-79.

2. Enrico Ammirati, Valentina Guicida, AzeemLalib et al. Determinants of outcome in patients with chronic ischemic left ventricular dysfunction undergone percutaneous intervention. BMC Cardiovasc Disorder 2015;15:137.

3. Daubert AMA, MassaroJ, Lioo L et all High risk percutaneous intervention is associated with reverse left ventricular remodeling and improved outcomes in patients with CAD and reduced ejection fraction, Am Heart J 2015,170(3):550-8.
4. Perera D,Stables R, Clayton T et along term mortality balloon pump assisted coronary intervention study(BCIS-1);A randomized controlled trial of elective balloon counterpulsation during high risk Percutaneous intervention Circulation 2013;127(2):207-12.

5. O. Neill WW, Kleimer NS, Moses J et al Prospective randomized clinical trial of haemodynamic support with Impella2.5 versus IABP in patients undergoing high risk percutaneous intervention, Circulation; the PROTECT II study, circulation 2012:126:1717-27. 